(2) The Author 2018. This is an Open Access article, distributed under the terms of the Creative Commons Attribution-

NonCommercial-NoDerivatives cence (http://creativecommon org/licenses/by-nc-nd/4.0/), which permits non-commercial re-use, distribution, and reproduction in any medium, provided the original work is unaltered and is properly cited. The written permission of Cambridge University Press must be obtaned University mercial re-use or in order to create a derivative work. the relevance of randomised controlled trials in evaluating some forms of psychological treatment (Lilienfeld et al, 2018). Despite special pleading from some quarters that ineffective treatments can be justified because they work for selected patients, Lilienfeld et al draw attention to the inglorious and lengthy history of psychiatric interventions that were subsequently proved to be useless or even harmful.

An introductory review article by Tyler and Kyriakopoulos considers the barriers to promoting EBP across the world. They point out the excellent work done by the World Health Organization in providing updated intervention guides for mental and substance misuse disorders in LMIC. In our second paper, Caneo and Calderon consider the emergence of EBP in Chile where, in recent years, there has been an increasing rapprochement between clinical psychologists and psychiatrists and their respective roles in the management of mental disorders (Moncada, 2008). Chile introduced a set of national guidelines for the detection and treatment of depression in 2004, followed by further guidelines for schizophrenia, bipolar disorder and alcohol and substance misuse, and these have been updated every few years. Nevertheless, the authors take the view that the concept of 'evidence' in Chile is still not understood by clinical professionals in any consistent way; the doctor- patient relationship is often considered more important than EBP.

In our third contribution to this theme, Samartzis et al consider the way in which substance misuse disorders are addressed in Cyprus. This is a relatively wealthy country but one which spends little on state aid for medical treatments. Opioid addiction and cannabis use are more prominent than cocaine and stimulant misuse, both of which have decreased in prevalence over the past decade. There is a move to provide a variety of evidence-based treatments for alcohol addiction as well as a publicly funded programme to support smoking cessation. Nevertheless, effectively treating addiction in the context of the Cypriot mental health system remains a challenge.

\section{Declaration of interest \\ None.}

\section{References}

Lilienfeld S. O., McKay D. \& Hollon S. D. (2018) Why randomised controlled trials of psychological treatments are still essential. The Lancet Psychiatry, http://dx.doi.org/10.1016/S2215-0366(18)30045-2.

Moncada L. (2008) The Psychotherapeutic Professions in Chile. http:// repositorio.uchile.cl/bitstream/handle/2250/121547/Moncada_Laura. pdf?sequence=1 (accessed 8 May 2018).

\title{
THEMATIC PAPER \\ Evidence-based practice in a multicultural world: changing with the times
}

\author{
Claire Tiley ${ }^{1}$ and Marinos Kyriakopoulos ${ }^{2,3,4}$
}

\section{${ }^{1}$ Core trainee in psychiatry, Maudsley Scheme, UK \\ ${ }^{2}$ Consultant child and adolescent psychiatrist, National and Specialist Acorn Lodge Inpatient Children's Unit, Child and Adolescent Mental Health Clinical Academic Group, South London and the Maudsley NHS Foundation Trust, London, UK \\ ${ }^{3}$ Visiting senior lecturer, Department of Child and Adolescent Psychiatry, Institute of Psychiatry, Psychology and Neuroscience, King's College London, London, UK; email mari- nos.kyriakopoulos@kcl.ac.uk ${ }^{4}$ Adjunct assistant professor, Department of Psychiatry, Icahn School of Medicine at Mount Sinai, New York, USA}

Keywords. Evidence-based prac tice; research capacity; sustainability; low- and middle-income countries.
Evidence-based practice (EBP), which is commonly implemented in high-income countries (HICs), integrates the best research evidence, clinical expertise and patient preferences in the planning and provision of healthcare for both physical and mental health conditions. Although the same principles of EBP apply in low- and middleincome countries (LMICs), research into and implementation of such interventions in these countries remains significantly behind compared with HICs. This article presents a brief overview of the global mental health agenda and initiatives aiming to address this pressing gap through the promotion of research and scaling up services, identification of barriers to developing and implementing EBP in LMICs, and possible solutions to overcome them.
Evidence-based practice (EBP) is the most common basis for making decisions related to patient care for the majority of physical and mental health conditions. It integrates the best research findings, clinical expertise and patient preferences in the planning and provision of healthcare. In high-income countries (HICs), evidence forms the foundation of clinical practice through guidelines, policies and health services infrastructure. However, there has been limited capacity in building up similar evidence in low- and middle-income countries (LMICs), and in evaluating its translational potential into sustainable structures and supporting its implementation through large-scale service changes (Eaton et al, 2018).

\section{The global picture}

The promotion of EBP in mental health across the world has been a very important development 

Open Access article, distributed under the terms of the Creative Commons Attribution-

NonCommercial-NoDerivatives licence (http://creativecommons. org/licenses/by-nc-nd/4.0/), which permits non-commercial re-use, distribution, and reproduction in any medium, provided the oriinal work is unaltered $c$ properly cited. The written permission of Cambridge University Press must be obtained for commercial re-use or in order to create a derivative work. of recent decades. The first significant milestone in this direction has been the landmark World Health Report 2001, Mental Health: New Understanding, New Hope (WHO, 2001), where the vision for a global mental health agenda came to the forefront. Subsequently, the identification of gaps in both research and service provision between HICs and LMICs was further pursued. It was highlighted that only $10 \%$ of the world's medical research addressed the health needs of the $90 \%$ of the global population residing in LMICs, with 3-6\% of mental health research in high-impact and indexed medical journals coming from these countries (Thornicroft et al, 2012). In addition, of the funding allocated to mental health research over a 10 -year period by two of the largest funders, the US National Institute of Mental Health (NIMH) and the UK Wellcome Trust, only 5\% was spent on global mental health (Chisholm et al, 2007). At the same time, large differences were identified in the numbers of mental health professionals between HICs and LMIC; for example, there were 6.6 psychiatrists per 100000 population in HICs, compared with fewer than 0.5 in low- and lower-middle-income countries, and more than 30 mental health nurses per 100000 population in HICs, compared with 0.4 in low-income countries, 2.5 in lower-middle-income countries and 7.1 in upper-middle income countries (WHO, 2015). This is translated in inequitable access to care, with $76-85 \%$ of patients in LMICs compared with $30-50 \%$ in HICs not receiving treatment for their mental health needs. The quality of treatment in LMICs is also likely to be poorer compared to that in HICs (WHO, 2013).

Several initiatives and large-scale international collaborations have emerged to accurately measure and work towards closing this gap. With the Lancet series on global mental health, the evidence base for scaling up of services worldwide has been given very high visibility (Chisholm et al, 2007). The Movement for Global Mental Health, a coalition of individuals and institutions committed to act in this direction, was launched in 2008 (Patel et al, 2011), while in the same year, the World Health Organization (WHO) Department of Mental Health and Substance Abuse launched the Mental Health Gap Action Programme (mhGAP). The mhGAP was aimed at scaling up services for mental, neurological and substance use disorders, especially in LMICs. It asserted that, with proper care, psychosocial assistance and medication, tens of millions could be treated for depression, schizophrenia and epilepsy, be prevented from suicide and begin to lead normal lives - even where resources are scarce (WHO, 2008). Two years later, it released the first version of its Intervention Guide for mental, neurological and substance use disorders in non-specialised health settings (WHO, 2010), which was subsequently updated following additional evidence, feedback and evaluation in 2016 (WHO, 2016). Both guides were informed by the limited but emerging evidence from LMICs and evidence from HICs, all adapted according to feasibility, acceptability to patients and availability of services in different counties. In parallel, the NIMH established the Collaborative Hubs for International Research on Mental Health, aiming to increase the evidence base for mental health interventions in LMICs through expansion of research activities with the goal of developing knowledge, tools, and sustainable research-based strategies which could be used by local stakeholders. The WHO Mental Health Action Plan 2013-2020 further highlighted the importance of strengthening information systems, evidence and research at a global level by including this as one of its four objectives (WHO, 2013).

A number of large research collaborations have also been particularly influential in building the evidence base for implementation of largescale interventions in LMICs. Currently active programmes include the PRogramme for Improving Mental health carE (PRIME), a consortium of research institutions working collaboratively with Ministries of Health in five countries (Ethiopia, India, Nepal, South Africa and Uganda), led from the University of Cape Town with partners in the UK and the WHO, and funded by the UK Department for International Development (Lund et al, 2012); and the Emerald (Emerging mental health systems in low- and middle-income countries) programme, a consortium consisting of 12 institutions from five European countries and six partners in Ethiopia, India, Nepal, Nigeria, South Africa and Uganda, funded by the European Commission under the 7th Framework Programme (Semrau et al, 2015). PRIME aims to develop the evidence base for scaling up integrated packages of care for priority mental disorders in primary and maternal healthcare settings, while Emerald targets health systems and seeks to identify barriers to, and solutions for, the scaled-up delivery of mental health services in LMICs.

\section{Barriers and solutions to developing and implementing EBP in LMICs}

One of the main issues affecting the development of evidence-based mental health practices, policies and systems in LMICs is the limited research specifically exploring the applicability and sustainability of such interventions in these countries. Although funding is indeed a significant constraint, capacity to conduct, manage, disseminate and apply high-quality research is also a key challenge, affected by weak research training, staffing limitations, lack of research culture and collaborations, and suboptimal infrastructure (Thornicroft et al, 2012). In addition, the implementation of EBP on a large scale in LMICs is related to a number of other barriers. The absence of mental health from the public health priority agenda has been suggested to be the 
greatest barrier of all (WHO, 2008), as the political will of decision makers is directly linked to financial resource allocation and development of services (Eaton et al, 2011). The organisation of mental health services, with their concentration around big cities and tertiary centres, and lack of integration with primary care, also hinders patient access, leads to isolation of patients from their families and wider community, and increases stigma (WHO, 2008; Eaton et al, 2011). Systems-related barriers, poor community participation and lack of ownership have also posed significant challenges to establishing integrated mental health service provision (Semrau et al, 2015). Finally, the lack of effective public health leadership has been identified as a major barrier in most LMICs (WHO, 2008).

As a result of all these large-scale research initiatives and collaborations, significant progress has been made not only towards identifying barriers to scaling up services in LMICs but also towards strategies to address these barriers. The commitment to promote, facilitate the implementation of, and scale up effective interventions has permeated the aims, objectives and principles of all these initiatives. Training local researchers, supporting institutions, advising on research infrastructure, devising collaborative projects, and addressing funding through investment in building research capacity are positive steps in this direction (Thornicroft et al, 2012). The unprecedented productivity of high-quality research papers in the past decade with the direct involvement of research institutions in LMICs is a testament to this outstanding effort. In addition, targeting local stakeholders is paramount to improving the care and delivery of EBP in LMICs. A number of stakeholders who take on responsibilities to scale up services for mental disorders in LMICs have been identified; these include governments, mental health groups, patients and their families, public health professional groups, social sector stakeholders, human right groups, United Nations agencies, research funding agencies and world banks including donor agencies (Chisholm et al, 2007). Sustainable strategies for integrated large-scale coverage through simple packages of care delivered in non-hospital settings, and broadening of the role of clinicians in planning, training and advising decision makers in their area of expertise will allow more people in LMICs to have access to mental healthcare (Eaton et al, 2011). Finally, the development of tools to assess local needs, systems and costs; strategies to engage decision makers; involvement of patients and their carers; feasible, fair and sustainable resourcing; and evaluation of processes and scaled-up interventions are all likely to assist with transformations in the mental health services of LMICs (Semrau et al, 2015).

\section{Conclusions}

Building research capacity tailored to the needs and practices of LIMCs and strategic promotion of EBP through collaboration, effective communication and systems changes are paramount in ensuring that all people across the world access the best clinical care. Further understanding of the barriers to developing, evaluating and sustaining EBP at a global level and addressing these barriers is urgently needed.

\section{References}

Chisholm D., Flisher A. J., Lund C., et al (2007) Scale up services for mental disorders: a call for action. Lancet, 370, 1241-1252.

Eaton J., Gureje O., De Silva M., et al (2018) A structured approach to integrating mental health services into primary care: development of the Mental Health Scale Up Nigeria intervention (mhSUN). International Journal of Mental Health Systems, 12, 11.

Eaton J., McCay L., Semrau M., et al (2011) Scale up of services for mental health in low-income and middle-income countries. Lancet, $378,1592-1603$

Lund C., Tomlinson M., De Silva M., et al (2012) PRIME: a programme to reduce the treatment gap for mental disorders in five low- and middle-income countries. PLoS Medicine, 9, e1001359.

Patel V., Collins P. Y., Copeland J., et al (2011) The movement for global mental health. The British Journal of Psychiatry, 198, 88-90.

Semrau M., Evans-Lacko S., Alem A., et al (2015) Strengthening mental health systems in low- and middle-income countries: the Emerald programme. BMC Medicine, 13, 79

Thornicroft G., Cooper S., Bortel T. V., et al (2012) Capacity building in global mental health research. Harvard Review of Psychiatry, 20, $13-24$.

WHO (2008) Mental Health Gap Action Programme (mhGAP): Scaling up Care for Mental, Neurological, and Substance Use Disorders. World Health Organization (http://apps.who.int/iris/bitstream/handle/10665/ 43809/9789241596206_eng.pdf?sequence=1, accessed 20 April 2018).

WHO (2010) Mental Health Gap Action Programme (mhGAP): Intervention Guide for Mental, Neurological and Substance Abuse Disorders. World Health Organization (http://apps.who.int/iris/ bitstream/handle/10665/44406/9789241548069_eng.pdf? sequence $=1$, accessed 20 April 2018)

WHO (2013) Mental Health Action Plan 2013-2020. World Health Organization (http://apps.who.int/iris/bitstream/handle/10665/ 89966/9789241506021_eng.pdf;jsessionid=FAED5FEA6CFF8CA7 F9CE54E116763EB0? sequence=1, accessed 20 April 2018).

WHO (2015) Mental Health Atlas 2014. World Health Organization (http://apps.who.int/iris/bitstream/handle/10665/178879/ 9789241565011_eng.pdf?sequence=1, accessed 20 April 2018).

WHO (2016) Mental Health Gap Action Programme (mhGAP): Intervention Guide 2.0 for Mental, Neurological and Substance Abuse Disorders. World Health Organization (http://apps.who.int/iris/ bitstream/handle/10665/250239/9789241549790-eng.pdf? sequence $=1$, accessed 20 April 2018) 